\title{
Bond Incident Degree Indices of Catacondensed Pentagonal Systems
}

\author{
Yongsheng Rao $\mathbb{D}^{1},{ }^{1}$ Adnan Aslam $\mathbb{D}^{2},{ }^{2}$ Muhammad Unfowan Noor, ${ }^{3}$ \\ A. Othman Almatroud, ${ }^{4}$ and Zehui Shao ${ }^{1}{ }^{1}$ \\ ${ }^{1}$ Institute of Computing Science and Technology, Guangzhou University, Guangzhou 510006, China \\ ${ }^{2}$ Department of Natural Sciences and Humanities, University of Engineering and Technology, Lahore, Pakistan \\ ${ }^{3}$ Knowledge Unit of Science, University of Management \& Technology, Sialkot 51310, Pakistan \\ ${ }^{4}$ Department of Mathematics, University of Hail, Hail, Saudi Arabia
}

Correspondence should be addressed to Zehui Shao; zshao@gzhu.edu.cn

Received 28 April 2020; Revised 14 July 2020; Accepted 25 July 2020; Published 20 August 2020

Academic Editor: Lucia Valentina Gambuzza

Copyright (c) 2020 Yongsheng Rao et al. This is an open access article distributed under the Creative Commons Attribution License, which permits unrestricted use, distribution, and reproduction in any medium, provided the original work is properly cited.

\begin{abstract}
The bond incident degree (BID) indices can be written as a linear combination of the number of edges $x_{i, j}$ with end vertices of degree $i$ and $j$. We introduce two transformations, namely, linearizing and unbranching, on catacondensed pentagonal systems and show that BID indices are monotone with respect to these transformations. We derive a general expression for calculating the BID indices of any catacondensed pentagonal system with a given number of pentagons, angular pentagons, and branched pentagons. Finally, we characterize the CPSs for which BID indices assume extremal values and compute their BID indices.
\end{abstract}

\section{Introduction}

A pentagonal system is a connected geometric figure obtained by concatenating congruent regular pentagons side to side in a plane in such a way that the figure divides the plane into one infinite (external) region and a number of finite (internal) regions, and all internal regions must be congruent regular pentagons. In a pentagonal system, two pentagons are adjacent if they share an edge. In this work, we consider only catacondensed pentagonal systems (CPSs), i.e., pentagonal systems which have no internal vertices. This is equivalent to say that pentagonal system has only pentagons of type $L_{1}, L_{2}, A_{2}$, and $A_{3}$ (see Figure 1). We will denote by $l_{1}, l_{2}, a_{2}$, and $a_{3}$, respectively, the number of $L_{1}, L_{2}, A_{2}$, and $A_{3}$ pentagons the CPS has. We will denote the set of all CPSs with $n$ pentagons by $\mathrm{CH}_{n}$. For more details on pentagonal systems and this theory, we refer the readers to [1]. We are interested in studying the behavior of general bond incident degree (BID) indices over pentagonal systems. For details about the pentagonal systems, see [2-7]. One of the much studied topological indices was put forward by the Randić index [8]. For a graph $G$, it is denoted by $R(G)$ and is defined as

$$
R(G)=\sum_{u v}\left(d_{u} d_{v}\right)^{-(1 / 2)},
$$

where $d_{u}$ denotes the degree of the vertex $u$ and sum runs over all edges $u v$ of $G$. There are many topological indices which appeared in the literature of mathematical chemistry (see for example [9-13]). Among the degree-based topological descriptors, the most studied are the first and second Zagreb indices [14-17], the sum-connectivity index [17-19], the atom-bond connectivity index $[17,20]$, the augmented Zagreb index [17, 21, 22], the geometric arithmetic index [23-25], and the harmonic index [19, 26, 27]. Each of these BID indices can be expressed [28] as

$$
\operatorname{BID}(G)=\sum_{1 \leq i \leq j \leq \Delta} \theta_{i, j} x_{i, j}, \quad \Delta \text { is the maximum degree of } G,
$$


where $x_{i, j}$ is the number of edges in $G$ with end vertices of degree $i$ and $j$ and $\theta_{i, j}$ is a finite sequence of nonnegative real numbers. For instance, if BID is the Randic index, then $\theta_{i, j}=(1 / \sqrt{i j})$. From now on, BID indices of any graph $G$ induced by the sequence $\theta_{i, j}$ are defined by equation (2). In case of CPS, we have only vertices of degree 2, 3, and 4; therefore the general BID indices over $\mathrm{CH}_{n}$ will be induced by a sequence $\left\{\theta_{22}, \theta_{23}, \theta_{24}, \theta_{33}, \theta_{34}\right\}$ of nonnegative real numbers:

$$
\operatorname{BID}(G)=\theta_{22} x_{22}+\theta_{23} x_{23}+\theta_{24} x_{24}+\theta_{33} x_{33}+\theta_{34} x_{34},
$$

for every $G \in \mathrm{CH}_{n}$. Now, following [29], we define two transformations on a CPS, called linearizing and unbranching transformations. We will show that BID indices are monotone with respect to these transformations. This will give us a reduction procedure to compute the BID indices of any CPS. Finally, the results obtained will be applied to find the minimal and maximal values of BID indices over $\mathrm{CH}_{n}$.

\section{Linearizing and Unbranching a Catacondensed Pentagonal System}

In this section, we define two transformations: the linearizing transformation $T_{1}$ and the unbranching transformation $T_{2}$, for the catacondensed pentagonal systems. In order to define these two transformations, we first recall the definition of coalescence of two graphs [30]. Suppose $G_{1}$ and $G_{2}$ are graphs with edges $u_{1} v_{1} \in E\left(G_{1}\right)$ and $u_{2} v_{2} \in E\left(G_{2}\right)$. The coalescence of $G_{1}$ and $G_{2}$ with respect to edges $u_{1} v_{1}$ and $u_{2} v_{2}$ denoted by $G$ is formed by identifying the edges $u_{1} v_{1}$ and $u_{2} v_{2}$ in the following way:

$$
V(G)=\left[V\left(G_{1}\right)-\left\{u_{1}, v_{1}\right\}\right] \cup\left[V\left(G_{2}\right)-\left\{u_{2}, v_{2}\right\}\right] \cup\{u, v\},
$$

and the two edges in $G$ are adjacent if

(i) These are adjacent in $G_{1}$ or $G_{2}$, or

(ii) One is $u$ and the other one is adjacent to $u_{1}$ in $G_{1}$ or to $u_{2}$ in $G_{2}$, or

(iii) One is $v$ and the other one is adjacent to $v_{1}$ in $G_{1}$ or $v_{2}$ in $G_{2}$, or

(iv) First one is $u$ and the second one is $v$

Now, we define the linearizing transformation on a catacondensed pentagonal system. Let $P_{1}$ be catacondensed pentagonal system with angular pentagon $P$ and subcatacondensed pentagonal systems $Q_{1}$ and $Q_{2}$ as shown in Figure 2. Let $P_{2}$ be the catacondensed pentagonal system obtained by applying coalescing operation on $P_{1}$ that is moving the catacondensed pentagonal systems $Q_{2}$ to the $2-2$ edge of angular pentagon $P$. We call the pentagonal system $P_{2}$ the linearization of pentagonal system $P_{1}$ at the pentagon $P$.

Let $\Theta_{1}=-\theta_{22}+2 \theta_{23}-2 \theta_{24}+3 \theta_{33}-2 \theta_{34}$; then, the difference of BID indices of the catacondensed pentagonal systems $P_{1}$ and $P_{2}$ is calculated in the next theorem.

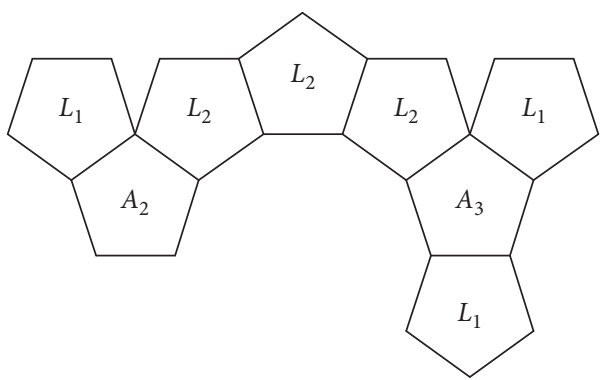

Figure 1: Types of pentagons.

Theorem 1. Let $P_{2}$ be a CPS obtained from $P_{1}$ by linearizing at angular pentagon $P$. Then,

$$
\operatorname{BID}\left(P_{2}\right)-\operatorname{BID}\left(P_{1}\right)=\Theta_{1} .
$$

Proof. In Figure 2, bold edges in $P_{1}$ and $P_{2}$ are the ones whose degree will change after the linearizing transformation. We collect all the bold edges in the set $E$ from $P_{1}$. Similarly, collect all the bold edges in the set $F$ from $P_{2}$. Note that the set of edges $E\left(P_{1}\right) \backslash E$ and $E\left(P_{2}\right) \backslash F$ is in one to one correspondence in such a way that for each edge in $E\left(P_{1}\right) \backslash E$, the degree of end vertices is equal to those of corresponding edge in $E\left(P_{2}\right) \backslash F$. Hence,

$$
\begin{aligned}
\operatorname{BID}\left(P_{2}\right)-\operatorname{BID}\left(P_{1}\right) & =4 \theta_{23}+3 \theta_{33}-\left(\theta_{22}+2 \theta_{23}+2 \theta_{24}+2 \theta_{34}\right) \\
& =-\theta_{22}+2 \theta_{23}-2 \theta_{24}+3 \theta_{33}-2 \theta_{34}=\Theta_{1} .
\end{aligned}
$$

If $\Theta_{1}<0$, then from above theorem, we have $\operatorname{BID}\left(P_{2}\right)<\operatorname{BID}\left(P_{1}\right)$. Hence, by applying a linearizing transformation, we can construct a new catacondensed pentagonal system whose BID indices are greater than the BID indices of original catacondensed pentagonal system. This fact is reflected in the next example.

Example 1. The sequence of CPS in Figure 3 satisfies

$$
\begin{aligned}
\operatorname{BID}\left(P_{1}\right) & >\operatorname{BID}\left(P_{2}\right)>\operatorname{BID}\left(P_{3}\right)>\operatorname{BID}\left(P_{4}\right) \\
& >\operatorname{BID}\left(P_{5}\right)>\operatorname{BID}\left(P_{6}\right),
\end{aligned}
$$

when $\Theta_{1}<0$.

Next, we define the unbranching transformation on catacondensed pentagonal system. Let $Z_{1}$ be CPS with branched pentagon $R$. Suppose that $Q_{2}$ is a pentagonal chain in $Z_{1}$. Note that such branched pentagon always exists in $Z_{1}$. Let $Z_{2}$ be a catacondensed pentagonal system obtained by applying coalescing operation on $Z_{1}$ that is moving the sub-catacondensed pentagonal system $Q_{3}$ to any of the 2-2 edge in the last pentagon of $Q_{2}$. This process is shown in Figure 4. Let $\Theta_{2}=\theta_{22}$ $-2 \theta_{23}+\theta_{33}$; then, the difference of BID indices of the catacondensed pentagonal system $Z_{1}$ and $Z_{2}$ can be expressed as the difference of $\Theta_{1}$ and $\Theta_{2}$. This is proved in the next theorem.

Theorem 2. Let $Z_{2}$ be a catacondensed pentagonal system obtained from $Z_{1}$ by unbranching at $R$; then, 


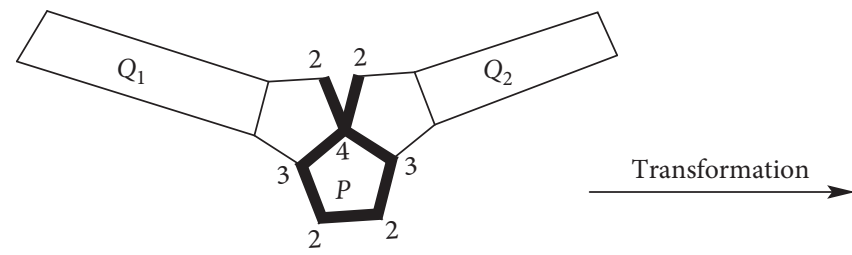

$P_{1}$

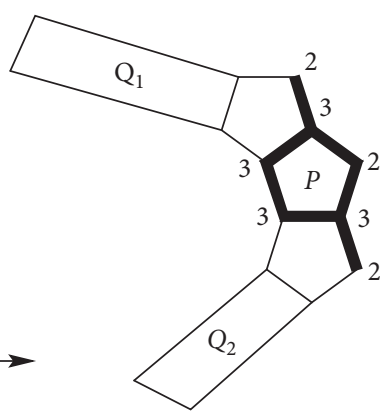

$P_{2}$

Figure 2: The linearization transformation $T_{1}$.

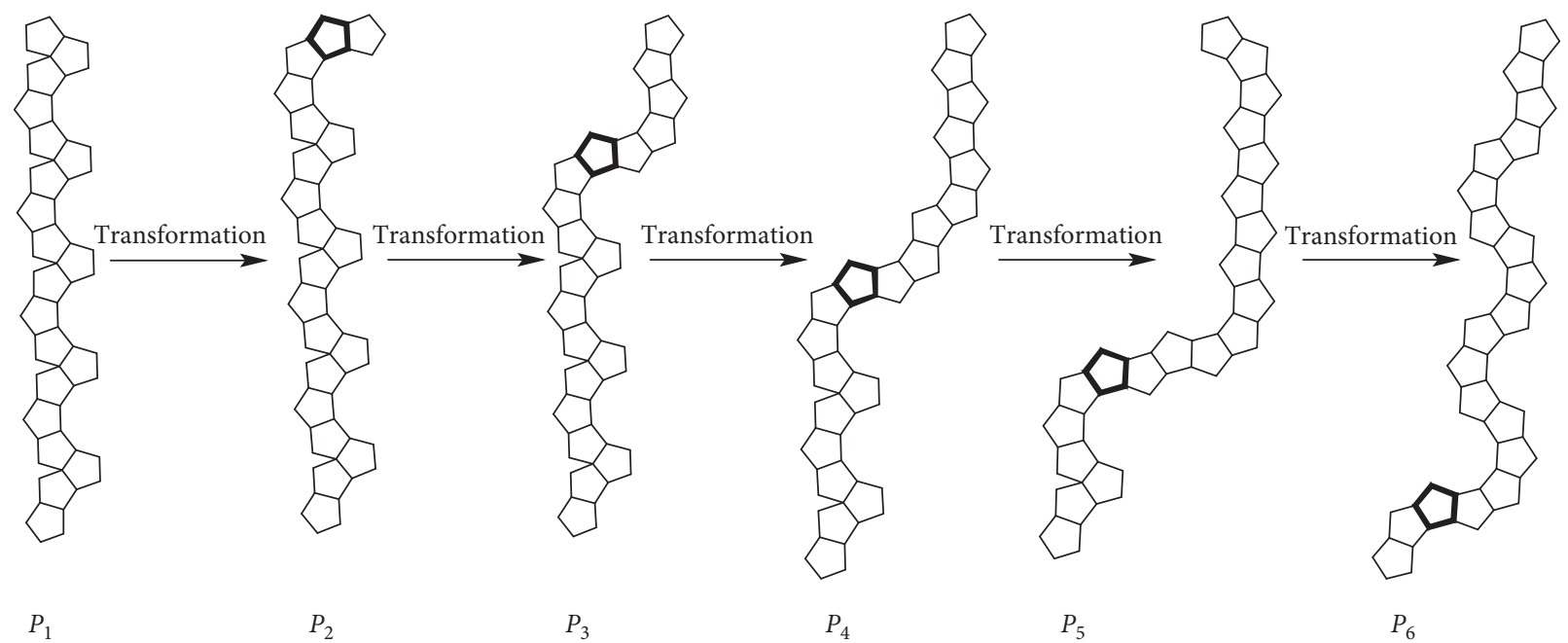

Figure 3: $\operatorname{BID}\left(P_{1}\right)>\operatorname{BID}\left(P_{2}\right)>\operatorname{BID}\left(P_{3}\right)>\operatorname{BID}\left(P_{4}\right)>\operatorname{BID}\left(P_{5}\right)>\operatorname{BID}\left(P_{6}\right)$.
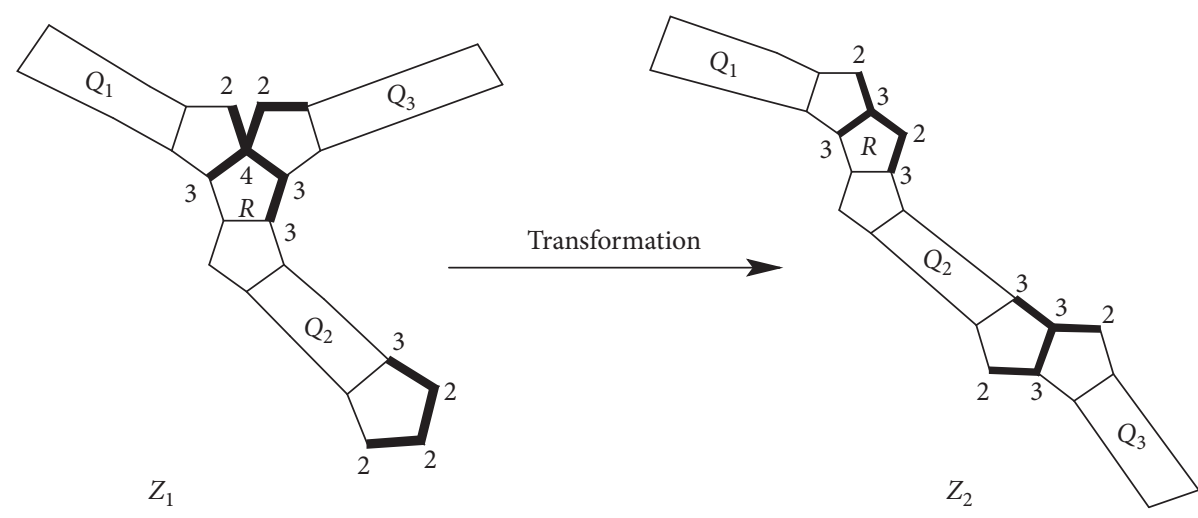

FIgURE 4: The unbranching transformation $T_{2}$.

$$
\operatorname{BID}\left(Z_{2}\right)-\operatorname{BID}\left(Z_{1}\right)=\Theta_{1}-\Theta_{2} .
$$

Proof. In Figure 4, bold edges in $Z_{1}$ and $Z_{2}$ are the ones whose degree will change after the unbranching transformation. We collect all the bold edges in the set $E$ from
$Z_{1}$. Similarly, collect all the bold edges in the set $F$ from $Z_{2}$. Note that the set of edges $E\left(Z_{1}\right) \backslash E$ and $E\left(Z_{2}\right) \backslash F$ is in one to one correspondence in such a way that for each edge in $E\left(Z_{1}\right) \backslash E$, the degree of end vertices is equal to those of the corresponding edge in $E\left(Z_{2}\right) \backslash F$. Hence, 


$$
\begin{aligned}
\operatorname{BID}\left(Z_{2}\right)-\operatorname{BID}\left(Z_{1}\right)= & 5 \theta_{23}+3 \theta_{33}-\left(2 \theta_{22}+\theta_{23}+2 \theta_{34}\right. \\
& \left.+2 \theta_{24}+\theta_{33}\right) \\
= & -2 \theta_{22}+4 \theta_{23}-2 \theta_{24}+2 \theta_{33}-2 \theta_{34} \\
= & \left(-\theta_{22}+2 \theta_{23}-2 \theta_{24}+3 \theta_{33}-2 \theta_{34}\right) \\
& -\left(\theta_{22}-2 \theta_{23}+\theta_{33}\right) \\
= & \Theta_{1}-\Theta_{2} .
\end{aligned}
$$

Note that if $\Theta_{1}-\Theta_{2}<0$, then each of the BID indices defined on the catacondensed pentagonal system is monotone decreasing when we apply unbranching transformation. Next example depicts this fact.

Example 2. The sequence of CPS in Figure 5 satisfies

$$
\operatorname{BID}\left(P_{1}\right)>\operatorname{BID}\left(P_{2}\right)>\operatorname{BID}\left(P_{3}\right)
$$

when $\Theta_{1}-\Theta_{2}<0$.

\section{Method to Compute BID Indices of Catacondensed Pentagonal System}

Examples 1 and 2 show that we can transform any catacondensed pentagonal system into linear pentagonal chain by successively applying linearizing and unbranching transformations. The number of steps depends on the number of angular pentagons $a_{2}$ and number of branched pentagons $a_{3}$. Theorems 1 and 2 show that we can find the exact value of topological indices after applying these transformations. Denote $L_{n}$ by a linear pentagonal chain with $n$ pentagons; then, the topological indices of $L_{n}$ can be computed by the following formula:

$$
\operatorname{BID}\left(L_{n}\right)=4 \theta_{22}+2 n \theta_{23}+(2 n-3) \theta_{33}
$$

Our next results show that once we know the number of angular pentagons $a_{2}$, the number of branched pentagons $a_{3}$, and the number of pentagons $n$ in a catacondensed pentagonal system, we can compute its topological indices.

Lemma 1. If $K$ is a pentagonal chain with $a_{2}$ angular pentagons, then

$$
\operatorname{BID}(K)=\operatorname{BID}\left(L_{n}\right)-a_{2} \Theta_{1} .
$$

Proof. We will prove it by induction on $a_{2}$. If $a_{2}=0$, then $K=L_{n}$ and the result follows. Suppose that the result holds valid for pentagonal chain with less than $a_{2}$ pentagons. Let $K$ be a pentagonal chain with $a_{2}>0$ angular pentagons and let $L$ be the pentagonal chain obtained by applying linearizing transformation on an angular pentagon $K_{1}$ of $K$. Then, by Theorem 1,

$$
\operatorname{BID}(L)-\operatorname{BID}(K)=\Theta_{1}
$$

Since the pentagonal chain $L$ has $a_{2}-1$ angular pentagons, then by induction hypothesis,

$$
\operatorname{BID}(L)=\mathrm{BID}_{n}-\left(a_{2}-1\right) \Theta_{1}
$$

Finally,

$$
\begin{aligned}
\operatorname{BID}(K) & =\operatorname{BID}(L)-\Theta_{1} \\
& =\operatorname{BID} L_{n}-\left(a_{2}-1\right) \Theta_{1}-\Theta_{1} \\
& =\operatorname{BID} L_{n}-a_{2} \Theta_{1} .
\end{aligned}
$$

Our next theorem generalizes Lemma 1 to any catacondensed pentagonal system.

Theorem 3. Let $M$ be a catacondensed pentagonal system with $n$ pentagons, $a_{2}$ angular pentagons, and $a_{3}$ branched pentagons; then,

$$
\operatorname{BID}(M)=\operatorname{BID} L_{n}-a_{2} \Theta_{1}-a_{3}\left(\Theta_{1}-\Theta_{2}\right) .
$$

Proof. We will prove it by induction on $a_{3}$. If $a_{3}=0$, then by Lemma 1, the result holds. Suppose that the result is true for catacondensed pentagonal systems with less than $a_{3}>0$ branched pentagons and let $M$ be a catacondensed pentagonal system with $a_{3}$ branched pentagons. By applying unbranching transformation at branch $M_{1}$ of $M$, we obtain a pentagonal system $N$ which has $a_{3}-1$ branched pentagons and $a_{2}$ angular pentagons. Hence, by Theorem 2 ,

$$
\operatorname{BID}(N)-\operatorname{BID}(M)=\Theta_{1}-\Theta_{2}
$$

By induction,

$$
\operatorname{BID}(N)=\operatorname{BID}\left(L_{n}\right)-a_{2} \Theta_{1}-\left(a_{3}-1\right)\left(\Theta_{1}-\Theta_{2}\right) .
$$

Hence,

$$
\begin{aligned}
\operatorname{BID}(M)= & \operatorname{BID}(N)-\Theta_{1}+\Theta_{2} \\
= & \operatorname{BID}\left(L_{n}\right)-a_{2} \Theta_{1}-\left(a_{3}-1\right)\left(\Theta_{1}-\Theta_{2}\right) \\
& -\Theta_{1}+\Theta_{2} \\
= & \operatorname{BID}\left(L_{n}\right)-a_{2} \Theta_{1}-a_{3}\left(\Theta_{1}-\Theta_{2}\right) .
\end{aligned}
$$

Next example details the computation of BID topological indices of any catacondensed pentagonal system using Theorem 3.

Example 3. We compute the topological indices of catacondensed pentagonal system $P_{1}$ shown in Figure 1 . It contains 1 angular pentagon, 1 branched pentagon, and in total 10 pentagons. Hence, by Theorem 3, we have

$$
\begin{aligned}
\operatorname{BID}\left(P_{1}\right)= & \operatorname{BID}\left(L_{10}\right)-\Theta_{1}-\left(\Theta_{1}-\Theta_{2}\right) \\
= & \operatorname{BID}\left(L_{10}\right)-2 \Theta_{1}+\Theta_{2} \\
= & \left(4 \theta_{22}+16 \theta_{23}+13 \theta_{33}\right) \\
& -2\left(-\theta_{22}+2 \theta_{23}-2 \theta_{24}+3 \theta_{33}-2 \theta_{34}\right) \\
& +\theta_{22}-2 \theta_{23}+\theta_{33} \\
= & 7 \theta_{22}+10 \theta_{23}+4 \theta_{24}+8 \theta_{33}+4 \theta_{34} .
\end{aligned}
$$

For example, if the BID topological index is the Randic index, then $\theta_{i j}=(1 / \sqrt{i j})$ and so 


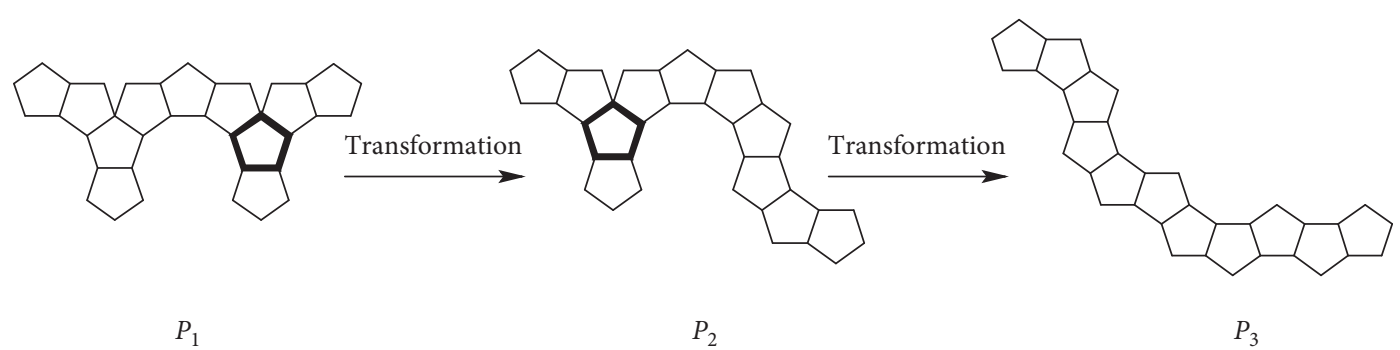

Figure 5: $\operatorname{BID}\left(P_{1}\right)>\operatorname{BID}\left(P_{2}\right)>\operatorname{BID}\left(P_{3}\right)$.

$$
R\left(P_{1}\right)=7 \frac{1}{2}+10 \frac{1}{\sqrt{6}}+4 \frac{1}{\sqrt{8}}+8 \frac{1}{3}+4 \frac{1}{\sqrt{12}}=14.7701 .
$$

If the BID index is the first Zagreb index $M_{1}\left(P_{1}\right)$, then $\theta_{i j}=i+j$, and

$$
M_{1}\left(P_{1}\right)=7 \times 4+10 \times 5+4 \times 8+8 \times 6+4 \times 7=186 .
$$

\section{Corollary 1.}

(i) If $\Theta_{1}<0$ and $\Theta_{1}-\Theta_{2}<0$, then the minimal value of BID indices over $C P_{n}$ is attained in $L_{n}$.

(ii) If $\Theta_{1}>0$ and $\Theta_{1}-\Theta_{2}>0$, then the maximal value of BID indices over $C P_{n}$ is attained in $L_{n}$.

Proof. Suppose that $\Theta_{1}<0$ and $\Theta_{1}-\Theta_{2}<0$; then, by Theorem 3 , the minimum value of BID indices is attained when $a_{2}=0$ and $a_{3}=0$. Hence, our catacondensed pentagonal system is a linear pentagonal system. Similarly, when $\Theta_{1}>0$ and $\Theta_{1}-\Theta_{1}>0$, then the maximum value is attained when $a_{2}=0$ and $a_{3}=0$ which occurs in the linear pentagonal system.

From Theorem 3, it is clear that the maximum and the minimum values of BID indices over $C P_{n}$ depend on the values of $a_{2}, a_{3}, \Theta_{1}$, and $\Theta_{2}$. Also, for any catacondensed pentagonal system $C P_{n}$ with $n \geq 2$, we have $l_{1} \geq 2$ and $0 \leq l_{2}, a_{2}, a_{3} \leq n-2$. Let $E_{n}$ and $F_{n}$ denote the CPSs with $n$ pentagons as shown in Figures 6 and 7.

\section{Corollary 2.}

(i) If $\Theta_{1}>0$ and $\Theta_{1}-\Theta_{2}<0$, then the minimal value of BID indices over $C P_{n}$ is attained in $E_{n}$.

(ii) If $\Theta_{1}>0$ and $\Theta_{1}-\Theta_{2}<0$, then the maximal value of BID indices over $C P_{n}$ is attained in $F_{n}$.

Proof

(i) Suppose that $\Theta_{1}>0$ and $\Theta_{1}-\Theta_{2}<0$; then, by Theorem 3 , the minimum value of BID indices is attained when $a_{3}=0$. Also, the maximum value of $a_{2}$ can be $n-2$ which occurs in $E_{n}$.

(ii) By Theorem 3, we get the maximum value of BID indices when $a_{2}=0$.

In a catacondensed pentagonal system, we have

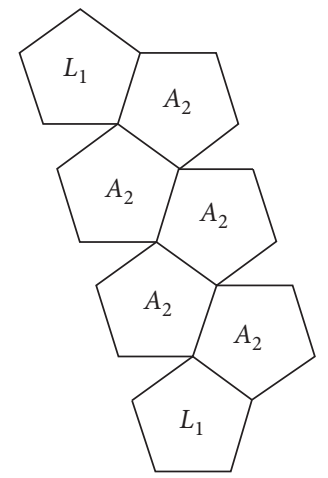

Figure 6: Catacondensed pentagonal system $E_{n}$ for $n=7$.

$$
\begin{aligned}
& l_{1}=a_{3}+2, \\
& n=a_{2}+l_{2}+a_{3}+l_{1} .
\end{aligned}
$$

We deduce

$$
a_{3}=\frac{1}{2}\left[n-\left(a_{2}+l_{2}+2\right)\right]=\frac{1}{2}\left[n-\left(l_{2}+2\right)\right] .
$$

If $n$ is even (odd), then the maximum value of $a_{3}$ will be obtained when $l_{2}=0\left(l_{2}=1\right)$ and this occurs in $F_{n}$.

\section{Corollary 3.}

(i) If $\Theta_{1}<0$ and $\Theta_{1}-\Theta_{2}>0$, then the minimal value of BID indices over $C P_{n}$ is attained in $F_{n}$.

(ii) If $\Theta_{1}<0$ and $\Theta_{1}-\Theta_{2}>0$, then the maximal value of $B I D$ indices over $C P_{n}$ is attained in $E_{n}$.

Example 4.

(i) For the second Zagreb index, we have $\theta_{i j}=i j$. Then, $\Theta_{1}=-5<0$ and $\Theta_{1}-\Theta_{2}=-6<0$. Hence, from Corollary 1 , the minimum value of BID indices is attained in $L_{n}$.

(ii) If BID indices are among the Randić, geometric arithmetic, harmonic, and sum-connectivity indices, then $\Theta_{1}>0$ and $\Theta_{1}-\Theta_{2}<0$. Hence, from Corollary 2 , the maximum value of BID indices is attained in $F_{n}$ and the minimum value is attained in $E_{n}$.

(iii) For the atom-bond connectivity index, we have $\theta_{i j}=\sqrt{(i+j-2) /(i+j)}$. In this case, $\Theta_{1}>0$ and 

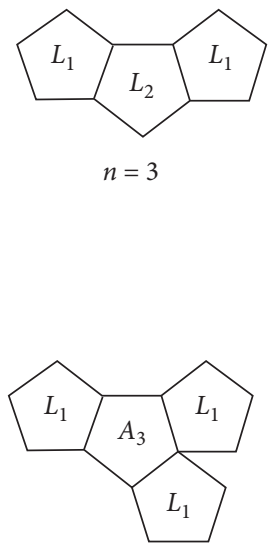

$n=4$
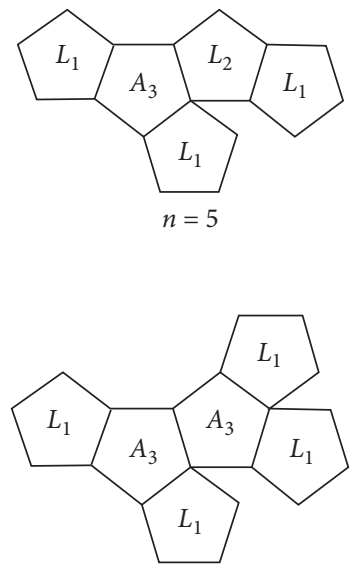

$n=6$
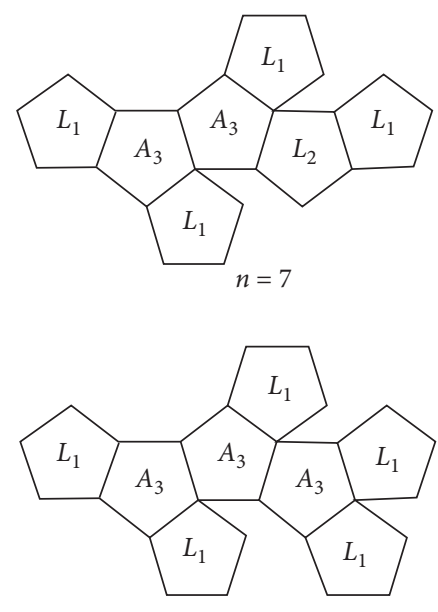

$n=8$

Figure 7: Catacondensed pentagonal system $F_{n}$ for $n=$ odd and $n=$ even.

TABle 1: Values of $\Theta_{1}$ and $\Theta_{2}$ for different BID indices.

\begin{tabular}{lcccccccc}
\hline BID indices & $i+j$ & $i j$ & $(1 / \sqrt{i j})$ & $(2 \sqrt{i j} /(i+j))$ & $(2 /(i+j))$ & $(1 / \sqrt{i+j})$ & $\left((i j)^{3} / \sqrt{(i+j-2)^{3}}\right)$ & $\sqrt{(i+j-2) /(i+j)}$ \\
\hline$\Theta_{1}$ & -2 & -5 & 0.032 & 0.0944 & 0.0619 & 0.0467 & -1.476 & 0.0019 \\
$\Theta_{2}$ & 0 & 1 & 0.168 & 0.404 & 0.333 & 0.138 & 3.3906 & -0.404 \\
Maximal & & & $E_{n}$ & $E_{n}$ & $E_{n}$ & $E_{n}$ & $L_{n}$ \\
Minimal & $L_{n}$ & $L_{n}$ & $F_{n}$ & $F_{n}$ & $F_{n}$ & $F_{n}$ & $L_{n}$ & \\
\hline
\end{tabular}

$\Theta_{1}-\Theta_{2}>0$; hence, from Corollary 1 , the maximum value of BID indices is attained in $L_{n}$.

In Table 1 , we have computed the values of $\Theta_{1}$ and $\Theta_{2}$ for different BID indices. This helps us to find the CPS which has the maximum and minimum values of the following BID indices.

\section{Concluding Remarks}

Among the well-known topological indices, there are various bond incident degree (BID) indices. In this paper, we have studied these BID indices for the catacondensed pentagonal systems and derived a general expression for calculating the BID indices of any catacondensed pentagonal system with a given number of pentagons, angular pentagons, and branched pentagons. We have also characterized the systems having maximum/minimum well-known BID indices from the class of all catacondensed pentagonal systems with a fixed number of pentagons. The present study can be extended in several directions. One of such directions is to study the general pentagonal systems for the BID indices. Also, there are many BID indices (see, for example, Table 1) for which we have not been able to characterize the systems having maximum/ minimum values from the class of all catacondensed pentagonal systems with a fixed number of pentagons.

\section{Data Availability}

No data were used to support the study.

\section{Conflicts of Interest}

The authors declare that they have no conflicts of interest.

\section{Acknowledgments}

This study was supported by the National Key R\&D Program of China (grant no. 2019YFA0706402) and the Natural Science Foundation of Guangdong Province (grant no. 2018A0303130115).

\section{References}

[1] A. Ali, Z. Raza, and A. A. Bhatti, "Extremal pentagonal chains with respect to bond incident degree indices," Canadian Journal of Chemistry, vol. 94, no. 10, pp. 870-876, 2016.

[2] I. Gutman, W. Yan, Y.-N. Yeh, and B.-Y. Yang, "Generalized Wiener indices of zigzagging pentachains," Journal of Mathematical Chemistry, vol. 42, no. 2, pp. 103-117, 2007.

[3] C. Xiao, H. Chen, and L. Liu, "Perfect matchings in random pentagonal chains," Journal of Mathematical Chemistry, vol. 55, no. 9, pp. 1878-1886, 2017.

[4] D. H. Rouvray, R. B. King, D. H. Rouvray, and R. B. King, "Harry in the limelight: the life and times of Harry Wiener," Topology in Chemistry, vol. 1, pp. 1-15, 2002.

[5] Y. Wang and W. W. Zhang, "Kirchhoff index of linear pentagonal chains," International Journal of Quantum Chemistry, vol. 110, pp. 1594-1604, 2018.

[6] H.-Y. Wang, J. Qin, and I. Gutman, "Wiener numbers of random pentagonal chains," Iranian Journal of Mathematical Chemistry, vol. 4, pp. 59-76, 2013.

[7] C. Xiao, H. Chen, and A. M. Raigorodskii, "A connection between the Kekule structures of pentagonal chains and the Hosoya index of caterpillar trees," Discrete Applied Mathematics, vol. 232, pp. 230-234, 2017.

[8] M. Randic, "Characterization of molecular branching," Journal of the American Chemical Society, vol. 97, no. 23, pp. 6609-6615, 1975. 
[9] B. Horoldagva and I. Gutman, "On some vertex degree based graph invariants," MATCH Communications in Mathematical and in Computer Chemistry, vol. 65, pp. 723-730, 2011.

[10] S. Hayat, M. Imran, and J. B. Liu, "Correlation between the Estrada index and p-electronic energies for benzenoid hydrocarbons with applications to boron nanotubes," International Journal of Quantum Chemistry, vol. 129, no. 23, 2019.

[11] M. Imran, M. K. Siddiqui, S. Ahmad, M. F. Hanif, M. H. Muhammad, and M. R. Farahani, "Topological properties of benzenoid, phenylenes and nanostar dendrimers," Journal of Discrete Mathematical Sciences and Cryptography, vol. 22, no. 7, pp. 1229-1248, 2019.

[12] E. Zogić and E. Glogic, "A note on the Laplacian resolvent energy, Kirchhoff index and their relations," Discrete Mathematics Letters, vol. 2, pp. 32-37, 2019.

[13] L. Z. Zhang and W. F. Tian, "Extremal catacondensed benzenoids," Journal of Mathematical Chemistry, vol. 34, no. 1, pp. 111-122, 2003.

[14] I. Gutman and N. Trinajstić, "Graph theory and molecular orbitals. Total $\varphi$-electron energy of alternant hydrocarbons," Chemical Physics Letters, vol. 17, no. 4, pp. 535-538, 1972.

[15] A. Ali, I. Gutman, E. Milovanović, and I. Milovanović, "Sum of powers of the degrees of graphs: extremal results and bounds," MATCH Communications in Mathematical and in Computer Chemistry, vol. 80, pp. 5-84, 2018.

[16] B. Borovićanin, K. C. Das, B. Furtula, and I. Gutman, "Bounds for Zagreb indices," MATCH Communications in Mathematical and in Computer Chemistry, vol. 78, pp. 17$100,2017$.

[17] I. Gutman, "Degree-based topological indices," Croatica Chemica Acta, vol. 86, no. 4, pp. 351-361, 2013.

[18] B. Zhou and N. Trinajstić, "On a novel connectivity index," Journal of Mathematical Chemistry, vol. 46, no. 4, pp. 12521270, 2009.

[19] A. Ali, L. Zhong, and I. Gutman, "Harmonic index and its generalizations: extremal results and bounds," MATCH Communications in Mathematical and in Computer Chemistry, vol. 81, pp. 249-311, 2019.

[20] E. Estrada, L. Torres, L. Rodrí Guez, and I. Gutman, “An atom bond connectivity index modeling the enthalpy of formation of alkanes," Indian Journal of Chemistry, vol. 37-A, pp. 849855, 1998

[21] B. Furtula, A. Graovac, and D. Vukičević, “Augmented Zagreb index," Journal of Mathematical Chemistry, vol. 48, no. 2, pp. 370-380, 2010.

[22] A. Ali, B. Furtula, I. Gutman, and D. Vukičević, "Augmented Zagreb index: extremal results and bounds," MATCH Communications in Mathematical and in Computer Chemistry, vol. 85, no. 2, 2021.

[23] D. Vukic̃ević and B. Furtula, "Topological index based on the ratios of geometrical and arithmetical means of end vertex degrees of edges," Journal of Mathematical Chemistry, vol. 46, pp. 1369-1376, 2009.

[24] K. C. Das, I. Gutman, and B. Furtula, "Survey on geometricarithmetic indices of graphs," MATCH Communications in Mathematical and in Computer Chemistry, vol. 65, pp. 595-644, 2011.

[25] A. Portilla, J. M. Rodríguez, and J. M. Sigarreta, "Recent lower bounds for geometric-arithmetic index," Discrete Mathematics Letters, vol. 1, pp. 59-82, 2019.

[26] L. Zhong, "The harmonic index for graphs," Applied Mathematics Letters, vol. 25, no. 3, pp. 561-566, 2012.

[27] S. Fajtlowicz, "On conjectures of Graffiti II," Congressus Numerantium, vol. 60, pp. 187-197, 1987.
[28] A. Ali, Z. Raza, and A. A. Bhatti, "Bond incident degree (BID) indices of polyomino chains: a unified approach," Applied Mathematics and Computation, vol. 287-288, pp. 28-37, 2016.

[29] J. Rada, R. Cruz, and I. Gutman, "Vertex-degree-based topological indices of catacondensed hexagonal systems," Chemical Physics Letters, vol. 572, pp. 154-157, 2013.

[30] J. Rada, "Energy ordering of catacondensed hexagonal systems," Discrete Applied Mathematics, vol. 145, no. 3, pp. 437-443, 2005. 